Results From the full study sample of 556 residents, 431 (78\%) individuals had both complete STI/HIV data and self-esteem assessment and were included in the analysis. The analysis population was $51 \%$ male and $49 \%$ female. Nearly $60 \%(n=254)$ had at least one STI/HIV. We observed no significant heterogeneity by gender in the effect of self-esteem on STI/HIV prevalence. Gender, transactional sex and alcohol use were retained as confounders in the final model; no other demographic or behavioural variables met our a priori statistical criteria for confounding. The adjusted prevalence ratios (PR) for STI/HIV for individuals with low self-esteem compared to those with medium self-esteem and high self-esteem were 0.95 (95\% CI 0.80 to 1.13 ) and 0.92 (95\% CI 0.67 to 1.27 ), respectively.

Conclusion Self-esteem was not independently associated with STI/ HIV prevalence in plantation residents in Tanzania. Our findings suggest that the role of self-esteem in reproductive health may be limited to its association with increased sexual risk behaviour, which subsequently affects STI/HIV prevalence. Interventions aimed at improving self-esteem may only be appropriate if they reduce likelihood of participation in risky behaviours.

\section{P1-S5.22 GENDER DIFFERENCES IN BEHAVIOURAL CORRELATES OF BIOLOGICALLY-CONFIRMED SEXUALLY TRANSMITTED INFECTIONS}

doi:10.1136/sextrans-2011-050108.200

A Berger, M Khan. University of Maryland, College Park, College Park, USA

Background Since sexually transmitted infection (STI) testing may not be feasible in all clinical and research settings, clinicians and researchers rely on self-reported sexual risk behaviours to serve as indicators of STI. The reported behaviours that are the strongest indicators of infection may differ for men and women.
Methods Using Wave III (2001-2002, young adulthood) of the National Longitudinal Study of Adolescent Health, we conducted analyses among white, black, and Hispanic participants $(\mathrm{N}=7185)$ to estimate gender-specific age-, race-, and poverty-adjusted associations between biologically-confirmed STI (gonorrhoea, chlamydia, and trichomoniasis) and indicators of sexual risk behaviour including young age of sexual initiation (less than 16 years), multiple (two or more) partnerships in the past year, lifetime number of partners, failure to use a condom at the most recent sex act, report of sex in the past year with an STI-infected partner, sex trade defined as paying or getting paid for sex, and report of past year diagnosis with gonorrhoea, chlamydia, and trichomoniasis, syphilis, herpes simplex virus, or HIV. We tested the significance and, when identified, report gender-specific race differences in the associations between sexual risk behaviour and STI.

Results Overall, most sexual risk behaviours were not good indicators of biologically confirmed STI. Identified correlates of infection differed for men and women. Among men, STI was associated with young age at first sex (adjusted OR 1.96, 95\% CI 1.27 to 3.01 ) and failure to use a condom at the most recent sex act (adjusted OR: $1.84,95 \%$ CI 1.12 to 3.01), though these indicators were not associated with STI among women. Among women, STI was moderately associated with multiple partnerships (adjusted OR: $1.64,95 \%$ CI 1.18 to 2.72 ) and lifetime number of partners (adjusted OR: $1.03,95 \%$ CI 1.01 to 1.06 ), though these were not STI indicators for men. Among white women only, report of sex with an STI-infected partner was associated with STI (adjusted OR: $2.22,95 \%$ CI 1.03 to 4.74 ). Indicators of sex trade and selfreported STI were not indicators biologically-confirmed STI for any population see Abstract P1-S5.22 Table 1.

Conclusions Indicators of sexual risk used in clinical and research settings should consider gender differences in markers of infection. Further, there is a need to identify more specific behavioural and social indicators of infection.

Abstract P1-S5.22 Table 1 Unadjusted and Adjusted ORs and 95\% Cls for Associations between Risky Sexual Behaviour and Biologically-Confirmed Chlamydia, Gonorrhoea, or Trichomoniasis among 7185 White, Black, and Hispanic Young Adults in the USA

\begin{tabular}{|c|c|c|c|c|}
\hline & \multicolumn{2}{|l|}{ Males $(\mathrm{N}=3252)$} & \multicolumn{2}{|l|}{ Females $(\mathrm{N}=3933)$} \\
\hline & Unadjusted OR (95\% CI) & Adjusted OR $(95 \% \mathrm{CI})^{*}$ & Unadjusted OR (95\% CI) & Adjusted OR (95\% CI)* \\
\hline \multicolumn{5}{|l|}{ Age of sexual initiation } \\
\hline Age at first sex $\dagger$ & 0.85 (0.78 to 0.92$)$ & $0.90(0.83$ to 1.00$)$ & $0.92(0.86$ to 0.99$)$ & $0.95(0.87$ to 1.03$)$ \\
\hline Sex younger than 16 & $2.47(1.64$ to 3.71$)$ & $1.96(1.27$ to 3.01$)$ & $1.30(0.91$ to 1.85$)$ & $1.26(0.86$ to 1.84$)$ \\
\hline \multicolumn{5}{|l|}{ Multiple partnerships } \\
\hline $2+$ partners in past year & $1.23(0.79$ to 1.91$)$ & $0.99(0.64$ to 1.55$)$ & 1.89 (0.38 to 2.58$)$ & 1.64 (1.18 to 2.72$)$ \\
\hline $4+$ partners in past year & $1.04(0.61$ to 1.77$)$ & $0.80(0.44$ to 1.44$)$ & $1.66(0.98$ to 2.81$)$ & $1.47(0.84$ to 2.57$)$ \\
\hline $6+$ partners in past year & $1.16(0.56$ to 2.41$)$ & $0.89(0.41$ to 1.94$)$ & $1.48(0.68$ to 3.21$)$ & $1.37(0.57$ to 3.31$)$ \\
\hline $8+$ partners in past year & $1.97(0.86$ to 4.48$)$ & $1.68(0.77$ to 3.67$)$ & $1.52(0.44$ to 5.24$)$ & $1.48(0.35$ to 6.32$)$ \\
\hline $10+$ partners in past year & 1.61 (0.56 to 4.62$)$ & $1.31(0.46$ to 3.78$)$ & $0.94(0.27$ to 3.24$)$ & $0.86(0.22$ to 3.30$)$ \\
\hline Total number of partners in past year $\dagger$ & $1.03(0.96$ to 1.11$)$ & $1.01(0.92$ to 1.10$)$ & 1.04 (0.97 to 1.09$)$ & 1.01 (0.96 to 1.07$)$ \\
\hline Lifetime number of partners $†$ & $1.02(1.00$ to 1.04$)$ & $1.01(0.99$ to 1.03$)$ & $1.03(1.01$ to 1.05$)$ & $1.03(1.01$ to 1.06$)$ \\
\hline \multicolumn{5}{|l|}{ Risky sexual partners } \\
\hline Sex with an infected partner in past year & $1.87(0.93$ to 3.77$)$ & $1.20(0.62$ to 2.31$)$ & $1.78(1.14$ to 2.79$)$ & $1.31(0.78$ to 2.20$)$ \\
\hline \multicolumn{5}{|l|}{ Condom Use Inconsistency } \\
\hline Condom use inconsistency in past year & $1.07(0.67$ to 1.70$)$ & $1.20(0.75$ to 1.92$)$ & $0.93(0.69$ to 1.26$)$ & $1.07(0.78$ to 1.46$)$ \\
\hline Non-condom use at most recent sex & $1.58(0.97$ to 2.57$)$ & $1.84(1.12$ to 3.01$)$ & $0.99(0.70$ to 1.40$)$ & $1.13(0.79$ to 1.62$)$ \\
\hline \multicolumn{5}{|l|}{ Sex trade } \\
\hline Ever paid for sex & $1.81(0.79$ to 4.18$)$ & $1.03(0.41$ to 2.55$)$ & $1.77(0.39$ to 8.07$)$ & $1.30(0.30$ to 5.62$)$ \\
\hline Ever got paid for sex & $1.69(0.74$ to 3.88$)$ & $0.94(0.38$ to 2.36$)$ & 2.95 (1.62 to 5.36$)$ & $1.34(0.70$ to 2.56$)$ \\
\hline Ever paid or got paid for sex & $1.76(0.92$ to 3.36$)$ & $1.04(0.50$ to 2.17$)$ & $2.73(1.50$ to 4.99$)$ & 1.32 (0.69 to 2.55$)$ \\
\hline \multicolumn{5}{|l|}{ Self-Reported STI } \\
\hline Self-reported STI in the prior year & $2.72(0.89$ to 5.81$)$ & $1.36(0.57$ to 3.28$)$ & 1.77 (1.08 to 2.89$)$ & $1.20(0.70$ to 2.06$)$ \\
\hline
\end{tabular}

${ }^{*}$ Controlling for age, poverty, and race/ethnicity.

†Continuous variable. 